\title{
Heuristic-based allocation of supply constrained blood platelets in emerging economies
}

\author{
Fredrik Ødegaard ${ }^{1}\left(\right.$ [) $\cdot$ Sudipendra Nath Roy ${ }^{1}$ (])
}

Received: 28 August 2020 / Revised: 28 August 2020 / Accepted: 16 April 2021 / Published online: 28 April 2021 (c) The Author(s), under exclusive licence to Springer Science+Business Media, LLC, part of Springer Nature 2021

\begin{abstract}
Platelets are valuable, but highly perishable, blood components used in the treatment of, among others, viral dengue fever, blood-related illness, and post-chemotherapy following cancer. Given the short shelf-life of 3-5 days and a highly volatile supply and demand pattern, platelet inventory allocation is a challenging task. This is especially prevalent in emerging economies where demand variability is more pronounced due to neglected tropical diseases, and a perpetual shortage of supply. The consequences of which have given rise to an illegal 'red market'. Motivated by experience at a regional hospital in India, we investigate the problem of platelet allocation among three priority-differentiated demand streams. Specifically we consider a central hospital which, in addition to internal emergency and non-emergency requests, faces external demand from local clinics. We analyze the platelet allocation decision from a social planner's perspective and propose an allocation heuristic based on revenue management (RM) principles. The objective is to maximize total social benefit in a highly supply-constrained environment. Using data from the aforementioned Indian hospital as a case study, we conduct a numerical simulation and sensitivity analysis to evaluate the allocation heuristic. The performance of the RM-based policy is evaluated against the current sequential first come, first serve policy and two fixed proportionbased rationing policies. It is shown that the RM-based policy overall dominates, serves patients with the highest medical urgency better, and can curtail patients' need to procure platelets from commercial sources.
\end{abstract}

Keywords Revenue management · Blood platelet · Resource allocation · Heuristics · Health care $\cdot$ Emerging economies

Fredrik Ødegaard

fodegaard@ivey.uwo.ca

1 Ivey Business School, Western University, London, ON, Canada 


\section{Introduction}

Platelets are an essential blood corpuscle that helps in blood clotting in case of injury and stops hemorrhage. Collected through blood banks and large hospitals, and separated from donated whole blood through processes like aphaeresis (Burgstaler 2006), platelets are often required for transfusion in post-therapeutic and replenishment treatment of various medical conditions, e.g. chemotherapy for oncological ailments viz. leukemia, multiple myeloma, aplastic anemia; bone marrow transplants; organ transplants; and radiation therapy (Whitaker and Sullivan 2005). In tropical, developing countries demand for platelets further surges with outbreaks of neglected, endemic diseases like dengue fever (Horstick et al. 2015), where platelet transfusion is one of the urgent medical treatments. This added source of uncertainty coupled with the fact that blood platelets have a shelf life of 3-5 days, the lowest among all blood components (Haijema et al. 2009; Zhou et al. 2011), results in platelet inventory allocation to be a persistent health care challenge in emerging economies. ${ }^{1}$ For instance, with sudden outbreaks of dengue fever, platelet demand can increase by five to tenfold in the Indian city of Bhopal and Mumbai (Jain 2016; Mishra 2014). In such situations, platelet price raise from 2 to 20 times the government determined maximum price (Chaterjee and Tiwari 2015; Kamath 2016; Jain 2016) and causes serious disturbances in the treatment of other diseases such as blood cancer (Shetty 2016).

As a result, in many emerging economies platelet transfusion often reflect de facto price and social discrimination. This is despite that most blood banks operate within the public health care system, or through regulated Red Cross subsidiaries, and strict governmental price regulations (Chaterjee and Tiwari 2015; Choudhury 2008, 2009; Ravishanker 2017). As demand far exceeds available inventory, patients have little option than to approach commercial bodies to privately procure platelets at premium prices. Similar trends are observed in other south-east Asian countries. For instance, in Pakistan price volatility may be even more extreme, and although the Pakistan Medical Association advocated for establishing a fixed price for platelets in the dengue prevalent city of Karachi (Dawn 2007), due to the severe supply-demand mismatch, a black market of blood and blood components has emerged. Blood touts reportedly collect blood forcibly from children (Singh 2009), impoverished migrant workers, or buy it cheap at $\$ 1$ per transfusion/unit from illegal donors (Raman 2008), and then sell it at illegal 'red markets' at prices as high as \$48 per unit (Anand 2015).

Motivated by the above serious health challenges facing developing countries in both normal and dengue outbreak situations, we analyze the effect of different platelet allocation policies. Specifically, we consider a regional hospital, with the capability to collect, store and transfuse blood platelets, that in addition provides support to local hospitals and health clinics with limited or no capacity to collect and extract platelets. The regional hospital has to decide how to allocate its limited supply of platelets among three stochastic daily demand streams: internal emergency, internal non-emergency, and external requests. The dynamic inventory stock at the regional hospital constitute platelets with different ages from zero to three days; where the

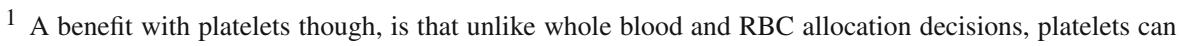
be assigned to patients irrespective of donor or recipient blood groups (Petraszko and Zeller 2018).
} 
initial day (0th day) is the processing day, and after the 3rd day platelets expire and are discarded. Contrary to the extant literature, but consistent with health care experience in emerging economies, supply of platelets is assumed to be stochastic and highly scarce-persistently lower than demand. In analyzing platelet allocation, we explore two important medical trade-offs: first, the policy consequences considering the medical priority of different patient segments; and second, the scarcity, consistent shortage and high perishability of platelets.

Paradoxically, although platelets are in persistent scarce supply, a significant wastage gets reported, including countries like India with extreme supply-demand mismatch (Debroy 2017). For instance, a study covering twelve hospitals in Iran reported high incidences of platelet wastage (Far et al. 2014); the Australia National Blood Authority, reported wastage of $16.5 \%$ of issued platelet compared to $3.3 \%$ for red blood cells, and $8.3 \%$ for fresh frozen plasma (National Blood Authority Australia 2013). The primary reasons for the high incident of platelet wastage is due to the very short shelf-life and that hospitals must ration the limited supply. Compared to other blood components, like red blood cells and blood plasma, platelets can essentially not be stored and hence lasts a much shorter time.

Platelet management in developing countries is not just a regional matter but also reflect international priorities. A case in point are the goals of the United Nations' 2030 Agenda for Sustainable Development (UN Resolution A/RES/71/313), which state that ensuring healthy living across the globe is a humanitarian need and top priority (United Nations Statistical Commission 2019). In a supply-constrained context, optimal platelet allocation to maximize total social benefit is arguably the first and most effective measure to resolve some of the issues. Furthermore, it can be argued that a robust platelet allocation policy might aid to reduce the supply-demand gap (Debroy 2017).

The remaining paper is organized as follows. Following a brief review of the extant literature, Sect. 2 provides the managerial and operational study context. Section 3 then presents the model, underlying assumptions, and a discussion on the intricate dependencies. Due to the inherent mathematical intractability, Sect. 4 provides and numerically illustrates a heuristic-based approach for platelet allocation. The heuristic, based on the Expected Marginal Seat Revenue-b algorithm from the Revenue Management (RM) literature, considers a step-wise decision process of rationing the platelets among the three priority-differentiated demand segments. Based on data from a regional hospital in India and through a simulation case study, Sect. 5 discusses the performance of the heuristic against three other policies: (i) sequential first come, first serve (currently the main policy implemented); (ii) a fixed fraction rationing; and (iii) a proportional demand fraction-based rationing. Two key metrics evaluated are proportion of demand satisfied and proportion of platelet wastage. Finally, Sect. 6 concludes the paper with a discussion on the implications and future research.

\subsection{Literature review}

Blood inventory management has gained much interest in both the operations management and operations research literature. Early work focused mainly on blood 
management as a perishable inventory, implementation of the derived results, and integration of the results in a decision support system for better functioning but with no specific focus on platelets, e.g. Prastacos (1984). Later work, including Haijema et al. (2005, 2007, 2009), stressed the short shelf-life of platelets, tried to capture orderup-to replenishment policies for inventory decisions, and aimed to minimize platelet wastage. Follow-up work include Zhou et al. (2011), who developed a detailed platelet inventory management model with two different ordering policies (normal and expedited); Abbasi and Hosseinifard (2014), who proposed FIFO policies for assigning age differentiated stock to the demand streams; and finally Civelek et al. (2015) introduced a protection ceiling while allocating platelet inventory for age differentiated demands. However, a predominant focus of the literature has been on classical inventory management approaches such as cost minimization Bertazzi et al. (2005) rather than allocation of inventories. Furthermore, anchored on ordering-up-to policies, the articles all rely on the crucial assumption that platelets are in abundant supply such that any order placed is fulfilled. Although this may approximate the reality (and luxury) of developed nations, for emerging economies this rarely if ever is the case, and hence the results regarding inventory ordering policies provide limited insight.

Optimal inventory allocation is less explored than traditional cost minimization inventory management problems especially when the inventory is perishable. Examples include Arslan et al. (2007), who discussed a continuous review heuristic for minimizing expected on-hand inventory by rationing a single product inventory between two demand differentiated classes based on their service level requirements in an infinite horizon; Wang et al. (2014), who considered two demand classes based on lead time (short and long); Frank et al. (2003), who analyzes a rationing policy based on periodic review policy among a stochastic demand class of lower priority and a deterministic demand class of higher priority; and Ding et al. (2016), who considering multiple Poisson distributed demand classes propose an inventory allocation policy considering partial class-dependent back-orders. The assumption that unfulfilled demand may be back ordered is common to all the cited papers. An exception is Frank et al. (2003) who, similar to us, study the problem in the context of lost sales. We stress that assuming supply to be in abundance or that unfulfilled demand may be back-ordered are not appropriate for our study context.

In an applied article, Ayvaz and Huh (2010) developed simple threshold heuristics in the allocation of hospital resources for two classes of patients one of which is back-ordered whereas other one is treated as lost sales. For perishable goods with backordering, Abouee-Mehrizi et al. (2019) developed an allocation policy for demand differentiated age of the stock, assuming periodic ordering and when ordered, inventory is fully replenished. In situations where supply matches demand, a natural consequence is that a fraction of the platelets will go to waste due to the inherent randomness of demand. Studies that focus on the trade-off between wasted (outdated) inventory and supply shortage, include (Blake et al. 2004, 2010), who advocated that a platelet ordering policy jointly considering outdated and shortage bounds better fulfill established service levels, and Duan and Liao (2013), who studies the effect of minimizing the expected platelet wastage for a given allowable shortage level. All three studies introduce age-based replenishment policies and emphasizes the age of the platelet inventory while inferring the performance of the suggested policy. 


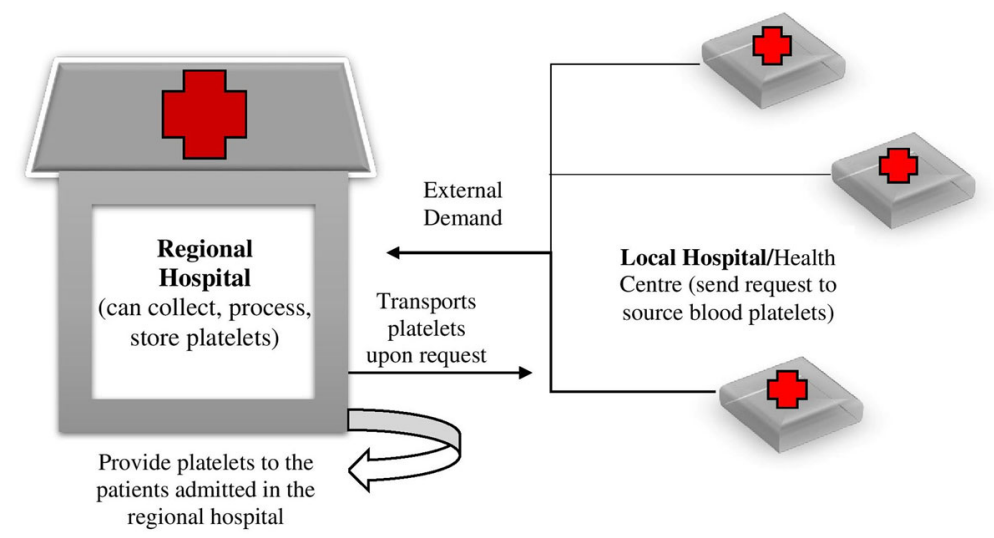

Fig. 1 Hospital network

Recently, Chen et al. (2019) argued that the demand for platelets is increasing and the supply of whole blood, from which platelets are produced, are declining in Hong Kong and Australia. They model the joint decision of blood collection and platelet production considering supply as a function of whole blood collection. Finally, Paul et al. (2019) considered the consequence of "over ordering" if down-stream players realize that supply is scarce and uncertain. They designed optimal contracts between regional blood banks and hospitals seeking platelets to stop this gaming phenomenon. Both articles acknowledge the fallibility of the assumption that supply of platelets is more than the demand, but still did not specifically address this in the inventory allocation decisions.

\section{Managerial health care context}

The motivation for this study stems from the platelet allocation practices at a 843 bed regional hospital in north-eastern India. The hospital, which has an average 137\% patient occupancy rate and serves patients from the north-eastern part of India, Nepal, Bhutan, and Bangladesh, has the capability to collect, store, and transfuse blood and blood platelets. ${ }^{2}$ In addition, the regional hospital supports a network of local hospitals and health clinics that only have the capability to transfuse platelets (but limited or no capability to collect, extract, and store platelets). Consequently, the regional hospital is responsible for allocating platelets to both internal and external requests. See Fig. 1.

Generally, in-patient physician visits start in the early morning (around $6 \mathrm{am}$ ), and once completed generates a list of patients requiring platelet transfusion and their urgency level for transfusion. This results in two internal daily demand streams:

\footnotetext{
2 Platelets are separated from donated blood by apheresis process to produce one transfusable platelet unit in a polymer bag. A transfusable platelet unit contains $3 \times 10^{11}$ platelets cells (Cushing 2013), and extracted either from blood of a single donor (single donor platelets, SDP) or from different blood units (random donor platelets, RDP) to pool the necessary amount of platelet cell count required to constitute one unit of transfusable platelets. In this paper, 'platelets' or 'platelet units' refer to one transfusable platelet unit.
} 
emergency and non-emergency patients. Both emergency and non-emergency demand grows throughout the day as new patients with various medical urgency needs arrive and in-patients' condition worsen (or improve). External demand requests from the local network hospitals usually arrive later (around 10-11 am), but is not categorized by urgency level. Ignoring political or geographical preferences, we assume the health administrator of the regional hospital gives no preference over any specific networked local hospital. Therefore rather than responding to individual requests from the local hospitals, we make a simplifying modification to only consider the total amount requested from all local networked hospitals. ${ }^{3}$

Around 8:30 am, a health administrator at the regional hospital has to decide on the daily platelet rationing and allocation for the three demand streams: the non-emergency patient demand which is already realized; the uncertain emergency demand which will eventually be realized in the latter part of the day; and the external demand for the networked local hospitals. The problem is that while demand for platelets is in abundance, supply is persistently scarce.

Every day the regional hospital receives a small amount of platelet from voluntary donors. However, this source is not sufficient and often doctors and hospital staff make unofficial improvisations and resort to a one-for-one policy, where patients in need of transfusion bring in their own donor (usually a family member or relative). Such ad hoc practices are possible because unlike blood transfusions, where blood type compatibility is imperative, platelet transfusion is generally compatible across blood types. A secondary "supply" of platelets occasionally occurs from unused and returned platelets from the local hospitals, and is known as a consignment scheme, see Fontaine et al. (2009) and Zhou et al. (2011). Unused platelets stems ironically from the scarce supply, due to a 'gaming of the system' where local hospitals overstate their need - knowing they will not get the amount requested; see Paul et al. (2019). In addition, local hospital will sometimes simultaneously requests from other sources, including private blood banks, non-governmental organizations, and donation from patients' family or relatives. In all cases, it may happen that a local hospital can fulfill their needs by less than what was provided by the regional hospital. In any event, every morning the regional hospital has a supply of age-differentiated stock of platelet units to allocate to the daily demand.

A complicating factor is that platelets are very perishable with a lifespan of about $3-$ 5 days (WHO 2005), which coupled with the stochastic, large demand and stochastic, small supply, makes platelet management a challenging task. The health administrator not only has to prioritize platelets allocation among the different daily demand streams, but also ration the limited supply over several days. If you allocate "too much" to less urgent demand streams, then you risk not being able to fulfill the more urgent demand streams. On the other hand, if you allocate "too little" (i.e. reserve "too much" for the more urgent), then you risk having platelet expire. The crux, as with all problems involving uncertainty, is that demand of the more urgent demand streams is realized post decision. Currently the hospital relies on a first come, first serve (FCFS) allocation policy, but with discretion in denying requests. In essence, the

\footnotetext{
3 The proposed model and allocation heuristic can be extended to include the allocation for the specific local health care requests.
} 


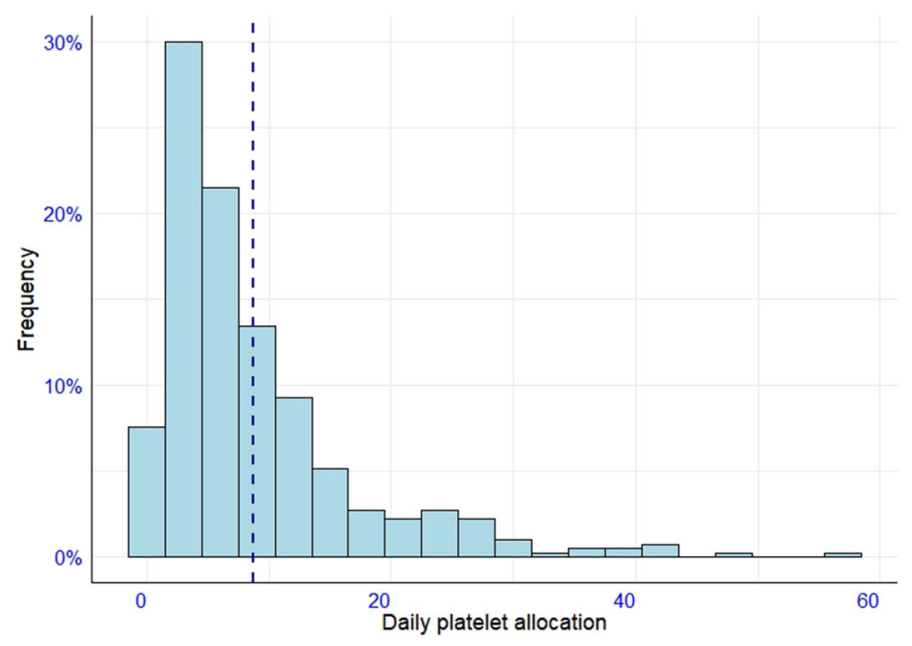

Fig. 2 Distribution of the aggregated daily platelet allocation

health administrator, using personal judgment and experience, reserves some amount for the later arriving emergency and external demand requests. However, this often leads to stock out situations for emergency patients who are medically critical at the time of need. Which in turn might stimulate the need to procure platelets from illegal 'red markets'. Henceforth, we refer to the FCFS platelet allocation policy as the 'sequential policy'.

The regional hospital provided daily platelet allocation data for over 1.5 years (from May 17, 2017 to Feb 15, 2019), which is shown in Fig. 2; the dashed vertical line intersecting the $x$-axis at 8.75, represents the average total daily allocation. As evident from the histogram the distribution is highly skewed with a median and third quartile of 6 and 11 platelet units, respectively. On the other hand, we do observe days with daily allocation of 20 to 40 , and even up to 60 units, which may seem counter-intuitive given the aforementioned highly supply-constrained environment. The 'long tail' represents rare days when organized blood donation drives coincide with the outbreak of, for instance, dengue fever. In these rare occasions, blood banks usually generate platelets from the entire collected blood volume in order to handle the dire situation caused by the outbreak. The duality of huge values with low frequency and much higher frequency of a fewer platelet allocation compared to mean bolsters the uncertain and challenging nature of platelet inventory allocation in emerging economies.

The objective of this paper is to propose an effective managerial policy of how much platelet to allocate to the three demand streams. However, of equal importance is that the policy is simple to communicate and implement. The policy is developed from the perspective of the health administrator who made a decision of daily platelet allocation for one realized and two uncertain demand streams with supply available in the regional hospital. As such, we seek a stationary policy providing fixed limits on the amount of platelet to allocate/reserve for these three different demand segments. We evaluate the proposed policy with the current first come, first serve allocation 
rule, and two fixed proportion-based rationing policies. The first one, allocates the total available platelet in accordance with a fixed proportion, e.g. emergency requests receive $50 \%$ of the available platelets, while non-emergency and external each receive $25 \%$ of the available platelets. The other fixed proportion policy allocates in proportion to the average request volume. Specifically, as the average emergency, non-emergency, and external requests represents $25 \%, 15 \%$, and $60 \%$, respectively, of the total daily demand, the allocation of the available platelets was assigned in the same proportions.

\section{Platelet allocation model framework}

Consider a hospital that makes daily decisions regarding the allocation of its available inventory of platelets. We index time by $t$ and consider an infinite planning horizon. Each day a random supply of fresh platelets, $S_{0}$, is generated; $S_{0}$ is drawn from the random Cumulative Distribution Function (CDF) $G(S)$, with corresponding density function $g(S)$. Each day platelets age and at the end of three days the platelets expire and have to be wasted. Denote $\mathbf{s}_{t}=\left[S_{0, t}, S_{1, t}, S_{2, t}, S_{3, t}\right]$ the vector of platelet inventory on day $t$; where $S_{0, t}, S_{1, t}, S_{2, t}$, and $S_{3, t}$, is the amount of zero, one, two, and three day old platelets, respectively. The total amount of platelets available on day $t$ is given by $S_{t}=S_{0, t}+S_{1, t}+S_{2, t}+S_{3, t}$. Although some physicians may have a preference for the more fresh platelet, we assume platelet inventory is depleted using a first in, first out rule, i.e. on any given day, 3 day old platelet inventory is allocated first, followed by 2 day old, followed by 1 day old, and lastly the fresh 0 day old platelet is used.

As we seek to promote an official policy we abstract away from the aforementioned one-for-one improvisation policy. This is motivated by trying to curtail the relevance of 'red market' and to find an efficient and equitable platelet allocation policies. Institutionalizing the one-for-one policy would further promote de facto socioeconomic discrimination and would therefore be morally and politically hard to justify. Furthermore, to simply the analysis we ignore the small portion of supply from platelets returned under consignment scheme of the local hospitals. However, in the numerical simulation analysis we include this secondary source of supply.

Each day demand for platelets stem from three sources, which arrive in increasing order of priority: first, the lowest priority non-emergency requests arrives, $D_{w}$; second, the middle priority of external requests arrive, $D_{x}$; and lastly the emergency requests arrives, $D_{y}$. See Fig. 3 . We assume $D_{y}, D_{x}, D_{w}$ are drawn from CDFs $F_{y}, F_{x}$, and $F_{w}$, respectively; with corresponding density functions $f_{y}, f_{x}, f_{w}$; finite means $\mu_{y}, \mu_{x}, \mu_{w}$; and finite variance $\sigma_{y}^{2}, \sigma_{x}^{2}, \sigma_{w}^{2}$.

The ensuing model formulation is based on continuous density functions (for both supply and demand), while the subsequent simulation analysis is based on discrete probability mass functions. For tractability, but with negligible abstraction from routine reality, we assume both the supply and demand processes are stationary. Although in reality demand may arrive in mixed order throughout the day, the health administrator has to decide the allocation at the start of each day-and crucially before total demand has been realized. As such, we assume the three demand streams arrive in increasing order of priority. Scarcity in supply as compared to demand is reflected by that total demand $D_{y}+D_{x}+D_{w}$ stochastically dominates daily supply $S_{0}$ (Table 1 ). 


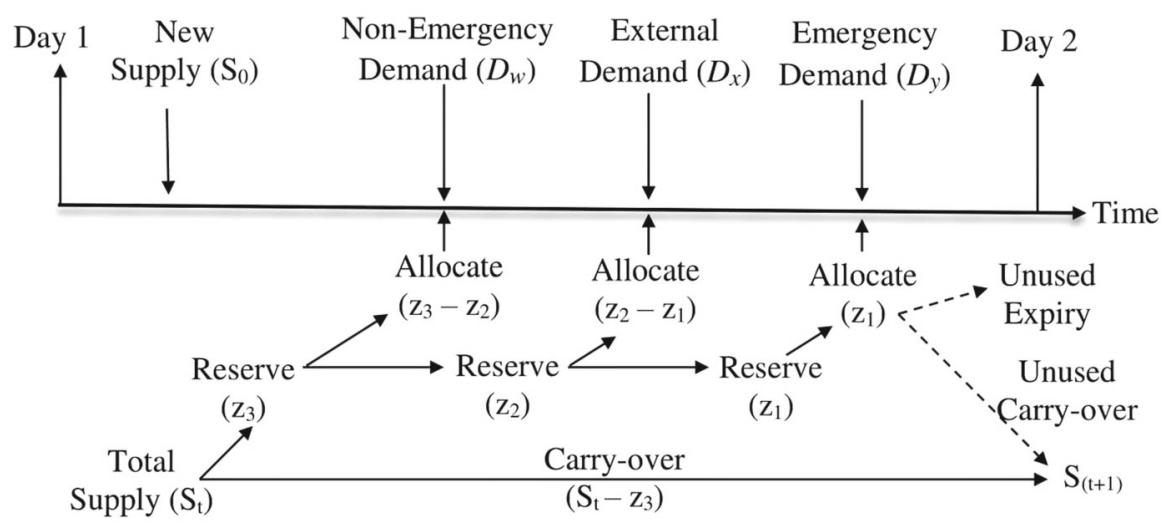

Fig. 3 Model framework for platelet allocation

Table 1 Notation

\begin{tabular}{ll}
\hline$p_{y}, p_{x}, p_{w}$ & Fulfillment benefit for emergency, external, non-emergency patient request \\
$\bar{p}$ & Weighted average fulfillment benefit for emergency and external patient \\
$\alpha_{x}, \alpha_{w}$ & Relative benefit parameter for external and non-emergency request \\
$D_{y}, D_{x}, D_{w}$ & Daily demand of emergency, external, non-emergency platelet requests \\
$F_{y}, F_{x}, F_{w}$ & Cumulative distribution function (CDF) of daily demand \\
$f_{y}, f_{x}, f_{w}$ & Probability density functions of daily demand \\
\hline
\end{tabular}

When a platelet request for an emergency, external, and non-emergency patient is fulfilled, we assume the regional hospital incurs an abstract-valued social benefit $p_{y}$, $p_{x}$, and $p_{w}$, respectively. While it may appear perverse to place a value on human health and discriminate between patients, from a social planner's perspective, and given the limited resources, a priority scheme among the different patient segments must be set. Therefore, to reflect the different medical urgency levels among the three streams, we assume $p_{w} \leq p_{x}<p_{y}$. In the subsequent analysis we standardize the rewards with respect to the emergency patients (i.e. $p_{y} \equiv 1$ ), and consider $p_{x}=\alpha_{x} \times p_{y}$, and $p_{w}=\alpha_{w} \times p_{y}$, where $0<\alpha_{w} \leq \alpha_{x}<1 .^{4}$

The task of the regional hospital health administrator is to decide on a stationary policy for allocating the total amount of available supply $S_{t}$ among the different demand streams, such that the total reward is maximized. With three demand classes this implies three nested quantities need to be set; the fourth quantity of how much to save for the remaining day is the amount left over. Let $z_{3}$ be the amount (out of $S_{t}$ ) to allocate to all three demand streams, $z_{2}$ the amount (out of $z_{3}$ ) to allocate to the external and internal emergency requests, and $z_{1}$ the amount (out of $z_{2}$ ) to allocate to the internal emergency requests; hence $\max \left\{S_{t}-z_{3}, 0\right\}$ is carried over to the following day. In other words, we seek a policy that sets a fixed amount to allocate (protect) to each of the three demand streams. However, since supply is scarce it might be that

\footnotetext{
4 Note that if there were no priority discrimination among the different streams, then the social planner should simply fulfill requests on a first come, first serve basis.
} 
there is not enough to cover the three quantities. On the other hand, if the realized demand for a lower priority patient group is less than the amount allocated, then the amount remaining is made available for the higher priority patient group.

We formulate the problem as a Markov Decision Process (MDP), with $V_{t}\left(\mathbf{s}_{t}\right)$ the optimal total value given a platelet inventory vector $\mathbf{s}_{t}$ in period $t$. The following Bellman equations summarizes the stochastic dynamics:

$$
\begin{aligned}
& V_{t}\left(\mathbf{s}_{t}\right)=\max _{\left(z_{3}, z_{2}, z_{1}\right)}\left\{( 1 - F _ { w } ( W ) ) \left[W p_{w}\right.\right. \\
& +\left(1-F_{x}(X)\right)\left[X p_{x}+\left[1-F_{y}(Y)\right]\left(Y p_{y}+\mathbb{E}_{S_{0}}\left[V_{t+1}\left(\mathbf{s}^{\prime}\right)\right]\right)\right. \\
& \left.+\int_{0}^{Y}\left(y p_{y}+\mathbb{E}_{S_{0}}\left[V_{t+1}\left(\mathbf{s}^{\prime \prime}\right)\right]\right) f_{y}(y) \delta y\right] \\
& +\int_{0}^{X}\left[x p_{x}+\left[1-F_{y}(X-x)\right]\left((X-x) p_{y}+\mathbb{E}_{S_{0}}\left[V_{t+1}\left(\mathbf{s}^{\prime}\right)\right]\right)\right. \\
& \left.+\int_{0}^{X-x}\left(y p_{y}+\mathbb{E}_{S_{0}}\left[V_{t+1}\left(\mathbf{s}^{\prime \prime \prime}\right)\right]\right) f_{y}(y) \delta y\right] f_{x}(x) \delta x \\
& +\int_{0}^{W}\left(w p_{w}+\left[1-F_{x}\left(S_{t}-w-z_{1}\right)\right]\left[\left(S_{t}-w-z_{1}\right) p_{x}\right.\right. \\
& +\left[1-F_{y}\left(z_{1}\right)\right]\left(z_{1} p_{y}+\mathbb{E}_{S_{0}}\left[V_{t+1}\left(\mathbf{s}^{\prime}\right)\right]\right) \\
& \left.+\int_{0}^{z_{1}}\left(y p_{y}+\mathbb{E}_{S_{0}}\left[V_{t+1}\left(\mathbf{s}^{\prime \prime}\right)\right]\right) f_{y}(y) \delta y\right] \\
& +\int_{0}^{S_{t}-w-z_{1}}\left[x p_{x}+\left[1-F_{y}\left(S_{t}-w-x\right)\right]\left(\left(S_{t}-w-x\right) p_{y}+\mathbb{E}_{S_{0}}\left[V_{t+1}\left(\mathbf{s}^{\prime}\right)\right]\right)\right. \\
& \left.\left.\left.+\int_{0}^{S_{t}-w-x}\left(y p_{y}+\mathbb{E}_{S_{0}}\left[V_{t+1}\left(\mathbf{s}^{\prime \prime \prime \prime}\right)\right]\right) f_{y}(y) \delta y\right] f_{x}(x) \delta x\right) f_{w}(w) \delta w\right\},
\end{aligned}
$$

where $W=\left(\min \left\{S_{t}, z_{3}\right\}-z_{2}\right)^{+} ; X=\left(\min \left\{S_{t}, z_{2}\right\}-z_{1}\right)^{+} ; Y=\min \left\{S_{t}, z_{1}\right\} ; \mathbf{s}^{\prime}=$ $\left[S_{0}, S_{1}^{\prime}, S_{2}^{\prime}, S_{3}^{\prime}\right]$, with, $S_{i}^{\prime}=S_{i-1}-\left(W+X+Y-\sum_{k=i+1}^{3} S_{k}\right)^{+} ; \mathbf{s}^{\prime \prime}=\left[S_{0}, S_{1}^{\prime \prime}, S_{2}^{\prime \prime}, S_{3}^{\prime \prime}\right]$, with, $S_{i}^{\prime \prime}=S_{i-1}-\left(W+X+y-\sum_{k=i+1}^{3} S_{k}\right)^{+} ; \mathbf{s}^{\prime \prime \prime}=\left[S_{0}, S_{1}^{\prime \prime \prime}, S_{2}^{\prime \prime \prime}, S_{3}^{\prime \prime \prime}\right]$, with, $S_{i}^{\prime \prime \prime}=S_{i-1}-\left(w+x+y-\sum_{k=i+1}^{3} S_{k}\right)^{+}$; the expectation $\mathbb{E}_{S_{0}}\left[V_{t+1}(\cdot)\right]$ is with respect to $\operatorname{CDF} G(S)$; and $(\cdot)^{+}=\max \{\cdot, 0\}$.

The above set of equations can be solved using standard value or policy iteration methods. However, with the non-linear nested 'max' and 'min' functions, and 
without additional highly simplifying assumptions on the stochastic elements, neither would yield closed-form solutions-or even closed-form expressions. Consequently, the Operations Research-oriented literature has mainly focused on establishing mathematical properties on the objective function and optimal solution, e.g. monotonicty, convexity, and submodularity of $V_{t}\left(\mathbf{s}_{t}\right)$, or threshold-type optimal policies. For the specific case given by (1), some of these properties are immediately intuitive. For instance, it is immediate that $V_{t}\left(\mathbf{s}_{t}\right)$ is increasing in $S_{0, t}, S_{1, t}, S_{2, t}, S_{3, t}$ (i.e. more supply is better). Other properties while equally intuitive may be more involved to prove, e.g. $z_{3}-z_{2}$ is decreasing in $p_{x}-p_{w}$ (i.e. as the reward between the external and non-emergency request increases, the amount to allocate to non-emergency request decreases).

While technical properties are interesting in their own right, our objective is to derive tangible, easy to solve, and managerially intuitive allocation policies. One of such implementable, easy to decipher, a managerial policy was described by Zhang et al. (2001) using spreadsheet modeling. Therefore, rather than focusing on the mathematical optimal solution to (1) we consider a heuristic-based approach.

\section{Heuristic-based platelet allocation policy}

Sharing many of the fundamental attributes of platelet management is the airline (capacity-based) revenue management problem; formerly 'yield management'. The problem facing airlines is how to allocate, for a specific flight on a specific date, the fixed seat capacity among the different fare classes it sells. Similar to platelets, seats on a flight are highly perishable: once a flight departs, empty seats are lost. Furthermore, the demand processes for the various fare classes are highly stochastic. A somewhat simplified dynamics though, is that in the airline context the supply remains fixed, i.e. the seat capacity usually does not change for specific routes. There are of course other differences but given the similarities, and the vast literature and development within revenue management, it is natural to see if some of the successful RM solutions would carry over to the platelet inventory allocation.

\subsection{Expected marginal platelet benefit allocation}

To draw on one the most prevalent and widely implemented RM solutions - the Expected Marginal Seat Revenue (EMSR) heuristic by Belobaba (1987), Belobaba (1989) - we impose two simplifications. First, we ignore the infinite time-horizon aspect to the problem and instead focus on a 'myopic' solution to the single day platelet allocation problem. In other words, we only consider the problem of allocating platelets to the three sources of daily demand, and do not consider the effect of carrying inventory over to the next day. One justification for this assumption is that allocating a platelet to an emergency patient today rather than tomorrow should not have any consequences. However, clearly, allocating a platelet to a non-emergency patient today at the expense of an emergency patient tomorrow would be worse. Second, and as a consequence of ignoring the effect of carrying over inventory, we only consider the 
two nested decisions of how much inventory to allocate for (i) the external and internal emergency demand $\left(z_{2}\right)$, and (ii) the internal emergency demand $\left(z_{1}\right)$; i.e. we do not consider the decision $z 3$, but instead make all $S_{t}-z_{2}$ available for the internal nonemergency requests.

To further simplify the exposition, but without loss of generality, we consider relative preference parameter $\alpha_{x}$ and $\alpha_{w}$ for the external patient and internal nonemergency patient requests, respectively. That is, we normalize the social benefit incurred for each platelet allocation with respect to the internal emergency patients, i.e. let $p_{y} \equiv 1$, and $p_{x}=\alpha_{x} \times p_{y}, p_{w}=\alpha_{w} \times p_{y}$, where $0<\alpha_{w} \leq \alpha_{x}<1$.

Following the logic of Littlewood's Two-Class model Littlewood (1972) and Belobaba (1987, 1989) EMSR-b heuristic, we consider the health administrator's platelet allocation in two nested backward induction steps. ${ }^{5}$ In the first step, the administrator decides $z_{1}$ quantity of platelets to reserve for internal emergency patients versus allocating them to the external requests. Next, in the second step, the health administrator reserves $z_{2}$ units of blood platelets to both external requests and internal emergency patients versus allocating them to the internal non-emergency requests. Thus, the external patients are assigned $z_{2}-z_{1}$, and the internal non-emergency patients are allocated $\left(S_{t}-z_{2}\right)^{+}$. Since the internal emergency patients' requirement is medically the most severe, followed by the external patients, this should maximize the overall social benefit.

We derive $z_{1}$ through the Littlewood (1972) Two-Class model—which is equivalent to the classic newsvendor solution. With both the internal emergency and external allocation rewards normalized to the internal emergency allocation, the critical fractile for internal emergency requests is given by,

$$
C_{y}=1-\alpha_{x}
$$

The critical fractile $C_{y}$ has a very intuitive and managerial relevant interpretation: it represents the proportion of time all internal emergency requests will be fulfilled. In other words, $1-C_{y}=\alpha_{x}$ is the chance that not all internal emergency requests can be fulfilled, which provides for a meaningful discussion point. We return to this in the conclusion. Based on the critical fractile the allocation for internal emergency requests is given by,

$$
z_{1}=F_{y}^{-1}\left(C_{y}\right)
$$

The amount of actual internal emergency requests fulfilled $(E R F)$ depends naturally on the emergency patient demand $\left(D_{y}\right)$, supply of platelets $\left(S_{t}\right)$, and how much was actually fulfilled for external $(X R F)$ and internal non-emergency $(N R F)$, and is given by,

$$
E R F=\min \left\{\max \left\{z_{1}, S_{t}-X R F-N R F\right\}, D_{y}\right\}
$$

\footnotetext{
5 Note that the EMSR-b is suitable for any $n$-class, non-overlapping, single-resource allocation problem (Belobaba 1989, 1992), and thus the heuristic could be expanded to multiple segments of external requests.
} 
If on a given day $t$, there are more emergency patient requests than the reserved or available amount, $D_{y}>\max \left\{z_{1}, S_{t}-X R F-N R F\right\}$, then the excess demand cannot be fulfilled and not backlogged, and thus simply lost. ${ }^{6}$ Next using the Belobaba (1987) EMSR-b approach, we first construct a weighted average platelet benefit for the external and internal emergency requests,

$$
\bar{p}=\frac{\mu_{y}+\alpha_{x} \mu_{x}}{\mu_{y}+\mu_{x}}
$$

Based on $\bar{p}$ an expression for the critical fractile $C_{x y}$, representing the external and internal emergency requests versus the internal non-emergency requests, is given by,

$$
C_{x y}=\frac{\bar{p}-\alpha_{w}}{\bar{p}}
$$

By substituting (5) we get,

$$
C_{x y}=1-\frac{\alpha_{w}\left(\mu_{y}+\mu_{x}\right)}{\mu_{y}+\alpha_{x} \mu_{x}}
$$

Based on the critical fractile $C_{x y}$ the total amount of platelet $z_{2}$ to be allocated for the two higher prioritized patient requests is given by,

$$
z_{2}=F_{x y}^{-1}\left(C_{x y}\right)
$$

where $F_{x y}$ is the distribution function with mean $\mu_{x y}$ for the total demand of external and internal emergency. Part of the appeal with EMSR-b, and source for its widespread implementation, is that if the two demand streams are independent and $F_{y}$ and $F_{x}$ belong to the same distribution family (e.g. normal, Poisson, etc.) then a functional form for $F_{x y}$ is easy to derive (e.g. normal, Poisson, etc.).

From $z_{2}$ the platelets to be reserved for external patient requests can be calculated by $z_{2}-z_{1}$. In other words, the health administrator can at most allocate $z_{2}-z_{1}$ platelets to the networked local hospitals. However, as above, the actual external request fulfillment $(X R F)$ depends on the demand $\left(D_{x}\right)$, supply $\left(S_{t}\right)$, the actual internal non-emergency fulfillment $(N R F)$, and is given by,

$$
X R F=\min \left\{\max \left\{S_{t}-N R F-z_{1}, 0\right\}, D_{x}\right\}
$$

where $N R F$, the actual non-emergency request fulfillment, is given by,

$$
N R F=\min \left\{\max \left\{S_{t}-z_{2}, 0\right\}, D_{x}\right\}
$$

\footnotetext{
6 In reality, the hospital informs family members or friends of the patient that they need to either secure their own donor; procure the platelets from the external sources; or look for any non-governmental organization which provides blood components as a community service.
} 
Note that for all three demand streams it may be that only partial fulfillment is provided. Hence, a key metric is the percentage of requests fulfillment for each of the three demand streams. The objective of the RM-based allocation policy is to maximize overall social benefit by allocating the platelets for the patients who need it the most, but at the same taking explicit consideration to the stochastic demand. We refer to the EMSR-based allocation policy as the 'RM policy'. Next we provide a numerical illustration based on data from the regional hospital.

\subsection{Numerical illustrations of RM-based policy}

Based on data provided by the regional hospital, the three daily demand streams were assessed to follow Poisson distributions; empirical support, albeit at the aggregate daily demand level, is shown in Fig. 2. Specifically, the Poisson parameter values for internal emergency, external and internal non-emergency patients were estimated at $\lambda_{y}=3, \lambda_{x}=7$, and $\lambda_{w}=2$, respectively; i.e. $F_{y}$ is Poisson(3), $F_{x}$ is Poisson(7), and $F_{w}$ is Poisson(2), which implies expected daily demands $\mu_{y}=3, \mu_{x}=7$, $\mu_{w}=2$. Assuming internal emergency and external requests are independent, the aggregate demand for both then follows a Poisson distribution with rate $\lambda_{x y}=10$; i.e. $F_{x y}$ is Poisson(10), with $\mu_{x y}=10$.

For the numerical illustration, we arbitrary set a base-case for the relative nonemergency preference parameter $\alpha_{w}=.33$, and consider the relative preference parameter of the external request $\alpha_{x}$ to range from .33 and .99 . This is motivated by that the external requests have higher priority than the internal non-emergency, but lower than the internal emergency requests. Based on Eqs. (3) and (8) above we can compute $z_{1}$ and $z_{2}$ as function of $\alpha_{x}$.

Figure 4 displays the allocation of platelets to internal emergency requests $\left(z_{1}\right.$; brown line), external requests $\left(z_{2}-z_{1}\right.$; blue line), and both internal emergency and external requests $\left(z_{2}\right.$; green line), as a function of the relative preference parameter of external requests $\alpha_{x}$. There are a couple of interesting observations.First, we see that the platelet units reserved for the internal emergency requests $\left(z_{1}\right)$ follow a decreasing step function, with the inflection points $\alpha_{x}=.35, .58, .80, .95$, from an initial 4 units allocated to a final 0 (i.e. no units are allocated for emergency requests when $\alpha_{x}>.95$. This is intuitive because, as the value for $\alpha_{x}$ increases, the relative social "benefit" of reserving platelets for emergency request diminishes, and the health administrator becomes indifferent between allocating to internal emergency and external requests. At the extreme when the relative importance of external patients $\alpha_{x}=1.0$, there is no difference between the emergency and external patients anymore.

Second, and more interestingly, we see that the allocation to emergency and external requests $\left(z_{2}\right)$ and only external requests $\left(z_{2}-z_{1}\right)$ are increasing. For $z_{2}$ (green line) the inflections occur at $\alpha_{x}=.43, .70$, and indicate a total allocation from 9 to 11 units. This implies that as the relative preference for external requests increases, the amount of platelets allocated to internal non-emergency decreases. And so while it is intuitive that the allocation to external requests $\left(z_{2}-z_{1}\right.$, blue line) is increasing in $\alpha_{x}$, the interesting feature is that this comes alternately at the expense of allocation to the internal emergency and non-emergency requests. 


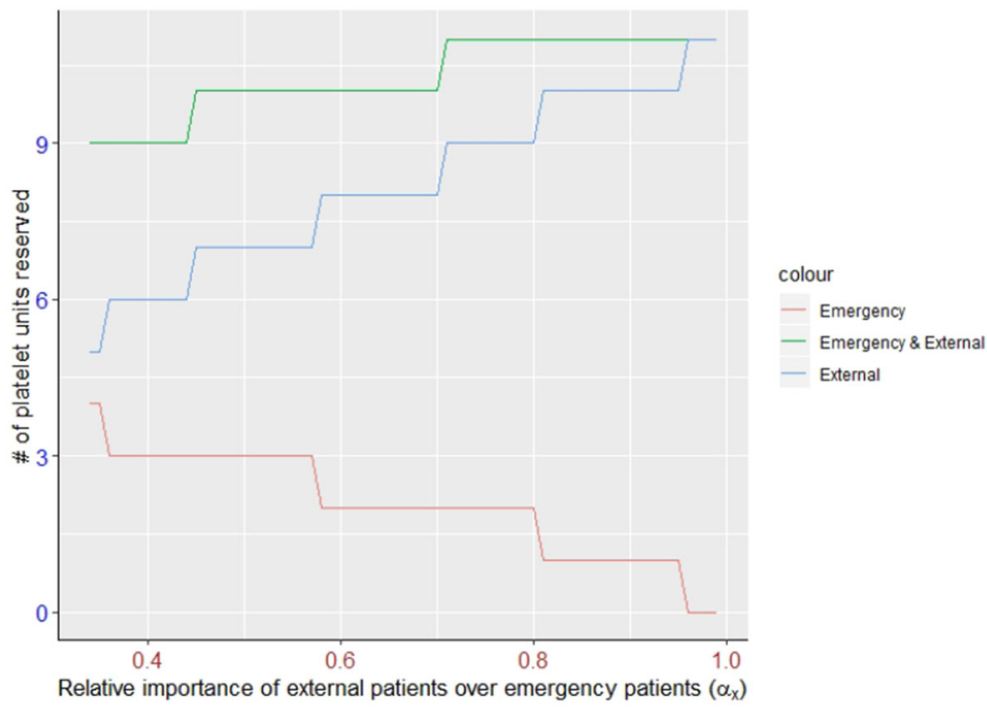

Fig. 4 Platelet allocation for internal emergency and external Patients

Finally, it is interesting to compare how the heuristic-based allocation compare to the expected value of the various demand streams. For instance, if $\alpha_{x}<.35$, then the allocation to internal emergency is more than the expected demand $\left(z_{1}>\mu_{y}\right)$, while if $\alpha_{x}>.58$, then the allocation is less than the expected demand $\left(z_{1}<\mu_{y}\right)$. In contrast, if $\alpha_{x}<.43$, then the allocation to internal emergency and external is less than the expected demand $\left(z_{2}<\mu_{2}\right)$, while if $\alpha_{x}>.70$, then the allocation is more than the expected demand $\left(z_{2}>\mu_{2}\right)$.

\subsubsection{Sensitivity analysis: varying $\alpha_{x}$ and $\alpha_{n}$}

The above numerical illustration was based on a fixed value of the relative nonemergency preference parameter $\alpha_{w}$. By varying $\alpha_{w}$ we can see what the interaction effect on the allocation amounts are. As we assume the relative importance of external requests $\left(\alpha_{x}\right)$ is more than the relative importance of non-emergency patients $\left(\alpha_{w}\right)$, we only consider values such that $\alpha_{w}<\alpha_{x}$. The objective of this illustration is to understand how the step-wise function that represents total platelet allocation for internal emergency and external requests $\left(z_{2}\right)$ behaves for each pair of values of $\alpha_{x}$ and $\alpha_{w}$.

Figure 5 displays the heat-map of platelet allocation to internal emergency and external patients as a function of $\alpha_{x}$ (x-axis) and $\alpha_{w}$ (y-axis). The left (a) panel is the entire heat-map for $\alpha_{x} \in[0,1]$, while the right (b) panel is the 'zoomed' area for $\alpha_{x} \in[.45, .55]$; for both panels the beige area above the 45 degree line (i.e. for $\alpha_{w}>\alpha_{x}$ ) represent non-permissible combinations. In both panels the darker the color the higher the value of $z_{2}$, while the lighter the color the lower the value.

We see that, for a given value of $\alpha_{w}$, by increasing $\alpha_{x}$, the colors get darker. Hence, indicating a higher allocation to internal emergency and external requests. In other words, for any horizontal 'cut' the allocation increases in $\alpha_{x}$, which is what we 

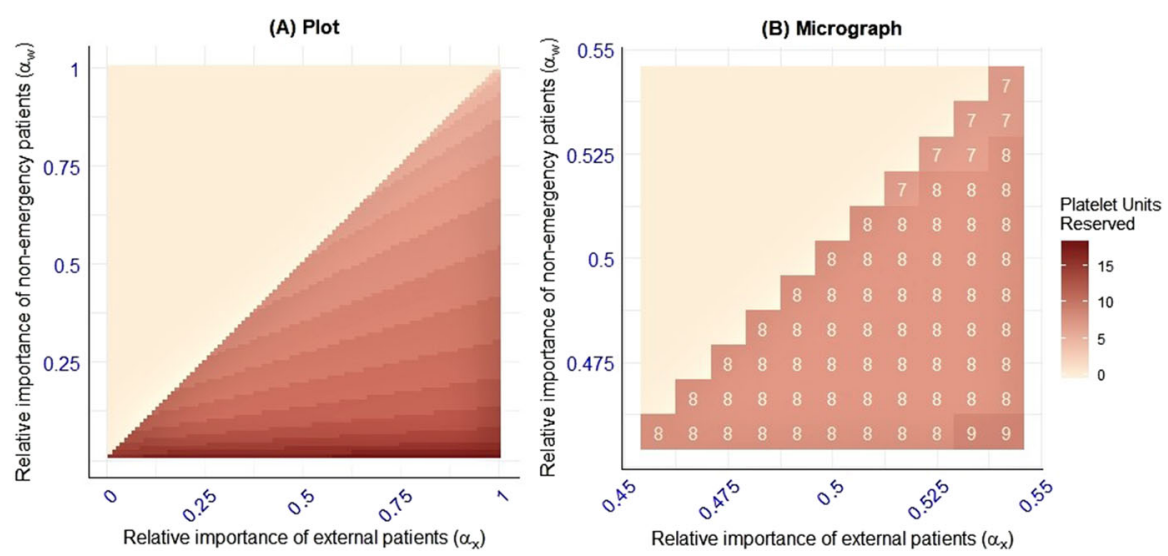

Fig. 5 a Plot and $\mathbf{b}$ micrograph of the reserved platelet units for emergency and external patients w.r.t $\left(\alpha_{x}, \alpha_{w}\right)$

observed above in the base-case. In contrast, for a given vertical 'cut' (for a given $\alpha_{x}$ ), the allocation is decreasing in $\alpha_{w}$. Both of these features gel with our expectations. Finally we see that as both $\alpha_{x}$ and $\alpha_{w}$ increases, the effect on $z_{2}$ is neither increasing nor decreasing - there is a non-monotone relationship depending on the inflection points that is passed. In other words, depending on the 'slope' of the increase in $\alpha_{x}$ and $\alpha_{w}$, the effect could be an increase or decrease in $z_{2}$. To further assess the effectiveness of the heuristic-based allocation policy we conducted an encompassing simulation study, which we discuss next.

\section{Effectiveness analysis of platelet allocation policies}

In this section, we discuss a comparative simulation analysis of the RM-based platelet allocation policy with two fixed proportion or fraction-based reservation policies, and the sequential FCFS policy the hospital currently employs. The purpose of each policy is to specify a dedicated amount to the three demand streams of the available platelet inventory. The simulation is based on the current processes and data as provided by the regional hospital in north-eastern India described earlier. Specifically, one of the authors spent considerable time interviewing various health administrators to understand the granular demand, supply, and return processes. The objective of the simulation analysis is to evaluate the four policies on three main performance metrics: (1) percent fulfilled request for each demand stream, (2) overall percentage of expired platelets, and (3) percent of days each demand stream is not $100 \%$ fulfilled.

\subsection{Simulation model framework}

A sequence of 300 days with random demand, supply, and returned platelets is generated, for which each allocation policy is employed to the available inventory. The simulation is repeated 1,000 times, where, to reduce variance in the evaluation, each 
policy is evaluated across the same realized sequence of 300 days. It is assumed that the three demand streams arrive in increasing order, and for all policies if realized demand is less than reserved supply, the remaining amount is made available to the next demand stream along-with the platelet units dedicated to it. Furthermore, at the end of the day, any left over platelets are carried over to the next day. Each demand stream is based on a Poisson distribution with the rate parameter as stated in the Sect. 4.2; i.e. mean requests from emergency $\mu_{y}$, external $\mu_{x}$, and non-emergency $\mu_{w}$ are 3,7 , and 2 , respectively.

The available inventory consists of platelets of different shelf life from zero (fresh) to three days old. Each day platelets remain in inventory they age by one day, and at the end of four days they are discarded. For all four policies, platelets are assigned as per the shelf life in the stock, i.e. older platelet are allocated first. The daily supply of fresh platelets is assumed to be drawn from a Poisson distribution with rate $\lambda_{s}=7$, i.e. on average there are 7 units fresh platelets provided each day. To account for returned stock from the consignment scheme, we incorporate a return process of two-day and three-day old platelets assuming Poisson distributions with rates $\lambda_{r 2}=1$ and $\lambda_{r 3}=2$; i.e. on average one two-day and two three-day old platelet units are returned. Thus, the total stock constitute the fresh platelets, returned stock, and any inventory carried over. We assume the fresh supply and return occur at the start of the day before any of the demand requests arrive.

The RM-based allocation policy is based on relative priority parameters $\alpha_{x}=.66$, and $\alpha_{w}=.33$, and kept constant through each simulation. The first fixed fraction allocation policy is where the health administrator reserves $50 \%$ of the daily supply for the internal emergency patients, $25 \%$ for the external demand requests and $25 \%$ to the internal non-emergency demands. The values were chosen arbitrarily but meant to reflect a priority scheme where external and internal non-emergency are weighted equally but half as compared to internal emergency requests. We label this policy as 'Reserve policy'. The second fraction-based policy is designed to reflect the priority based on the average demand levels $\mu_{y}, \mu_{x}$ and $\mu_{w}$. Specifically, since the average daily demand for emergency, external, and non-emergency were 3, 7, and 2, respectively, the policy is to allocate $25 \%(=3 / 12), 60 \%(\approx 7 / 12)$, and $15 \%(\approx 2 / 12)$ for emergency, external and non-emergency requests, respectively. This second fraction-based policy is labeled as 'Proportionate policy'.

To evaluate the policies, we let the simulation warm-up period be 200 days, and then measure each policy based on the final 100 days; this was done to remove biases and stabilize the simulation (Law and Kelton 2000). Also, we incorporated a 10 day period of peak dengue outbreak period in these 100 where total demand increase by five times (total daily demand is 60 units) and total supply also increase by five times due to coincidence of voluntary donation drives (in reality conducted by various non-governmental organizations, local clubs and medical awareness organizations) to compensate the huge shortfall faced by regional hospital; in such situation total daily supply reach to 50 units.

The first performance metric we consider is the percent of fulfillment for each individual demand segment, as well as the aggregate demand fulfillment across the three segments. Motivated by the high supply constraint environment, and that inventory reserved for emergency and external requests must not significantly increase platelet 
wastage, we also consider the percentage of platelet expiration. Through the discussions with the health administrators it seems that the main argument for the current sequential FCFS allocation policy is a general reticence to reserve platelet for potential requests that may not materialize, and hence have platelets wasted due to age-related expiry. As a social planner, it would be a moral setback if the platelet allocation policy ends up significantly increasing the expiry. Thus we considered 'expiry' as the second performance measure. Third, we measured the proportion of days the regional hospital failed to satisfy $100 \%$ of the respective demand requests.

As an overall measurement for percent of fulfillment we considered the average values (over the 1,000 simulation runs) across the final 100 days. For percentage of expiry, we calculated the average (over the 1,000 simulation runs) by considering only 90 days and removing the 10 day outbreak period. The reason for this is due to extreme scarcity of platelets during the 10 days, which severely lowers the average per day expiry. The simulation results, measured in percent, are tabulated in Table 2 and discussed in the next subsection. We also performed pair-wise two-sample t-test of the performance measures between the three new policies and the current sequential allocation policy. The results of the t-test signify if the differences between each new policy and the current sequential policy were statistically significant at the 5\% level; in which case the results are marked by a ' $*$ '.

\subsubsection{Model validation}

The simulation model was validated by comparing the output results with the real data of the regional hospital (which allocate platelets in essence by the sequential policy). Through discussions with the health administrators, it was revealed that the hospital generally satisfies $85-90 \%$ of the total daily demand; consistent with the performance of the simulation model. This 'black box' validation, by comparing real performance figures with model output measure data, ensures the reliability of the simulation model (Fletcher and Worthington 2009). Unfortunately, the hospital does not keep detail records of the other daily performance metrics, and as such the validation for the other metrics were through discussion and qualitative assessment.

\subsection{Results and analysis}

In Table 2 we see that, at an aggregate level, the total demand fulfillment was essentially the same across all four policies, and within the 85-90\% service level the regional hospital presently operates at. In particular, observe that total demand fulfillment was reduced by only $.67 \%$-points for the RM policy compared to the Sequential policy, which although significant at the $5 \%$ level, would be considered small and compensated by the segment specific fulfillment metrics. The Reserve and Proportionate policies also resulted statistically significant yet similar decrease in total demand fulfilled.

Through discussions with the hospital administrators we learned that they would be apprehensive to platelet allocation (i.e. reservation policy) that significantly increase waste through expiry. Intuitively, reserving platelets should engender an increase in expiry of platelet units, which indeed is reflected by the results. We see that the expiry 
Table 2 Performance measures (\%) of the four allocation policies

\begin{tabular}{lccll}
\hline & RM & Reserve & Proportionate & Sequential \\
\hline Demand fulfillment & & & & \\
Total (aggregated level) & $85.17^{*}$ & $85.17^{*}$ & $85.40^{*}$ & 85.84 \\
Emergency & $85.62^{*}$ & $99.51^{*}$ & $81.24^{*}$ & 62.48 \\
External & $92.70^{*}$ & $77.73^{*}$ & $93.35^{*}$ & 91.66 \\
Non-emergency & $70.51^{*}$ & $96.97^{*}$ & $80.76^{*}$ & 99.93 \\
Expiry & & & & \\
& 0.19 & $0.43^{*}$ & $0.29^{*}$ & 0.15 \\
Proportion days fail fulfill all requests & & & \\
Emergency & $29.21^{*}$ & $1.80^{*}$ & $34.49^{*}$ & 46.68 \\
External & $24.78^{*}$ & $55.47^{*}$ & $25.56^{*}$ & 23.90 \\
Non-emergency & $33.89^{*}$ & $8.69^{*}$ & $39.78^{*}$ & 0.20 \\
\hline
\end{tabular}

Two sample t-test w.r.t FCFS policy; *significant at $p>.05$

rate for the RM, Reserve, and Proportionate allocation policies were .19\%, .43\%, and $.29 \%$, respectively. Note though that compared to the rate of $.15 \%$ for the sequential, the increase in expiry was only statistically significant for the Reserve and Proportionate policies, and statistically insignificant for the RM policy. This suggests that even in a supply-constrained environment, it is possible to reserve platelets for the higher priority requests without compromising social responsibility, such as waste due to age-related expiry. However, there has to be a careful design and evaluated trade-off in how platelets are reserved.

If we delve deeper into the fulfillment across each individual demand streams we note some interesting features. First, we observe that compared to the Sequential policy the difference in demand fulfillment were all statistically significant. Second, no single policy dominated across all demand segments. This implies that there are tradeoffs between them with respect to the fulfillment of each segment. For instance, by comparing the Reserve, Proportionate, and Sequential policies, we see that they each favor a different demand stream; namely, internal emergency, external, and internal non-emergency, respectively. For the RM policy, while it did not have a fulfillment rate that dominated on any specific demand segment, it was overall more balanced but had the worst performance for internal non-emergency. On the other hand, note that the RM policy's $92.7 \%$ fulfillment of emergency is better than the sequential policy's $91.6 \%$ fulfillment for external requests. And so comparing the RM to the Sequential policy, the boost to a fulfillment of $85.6 \%$ for emergency requests (from 62.5\%) would arguably more than compensate the drop to $70.5 \%$ for non-emergency (from $99.9 \%$ ). Furthermore, since fulfillment of external requests, which the RM policy outperformed the Sequential policy on, is crucial for the centralized role of the regional hospital, we consider the RM policy to dominate the Sequential policy.

Again, however, depending on the socio-economic or health-political priorities, each policy has pros and cons and choosing one involves trade-offs. To illustrate consider the Reserve policy, which catered well to both non-emergency and emergency 
patients but drastically under-performed for the external requests. A 13.93\%-points and $14.97 \%$-points reduction of external demand fulfillment is observed when Reserve policy was evaluated against Sequential and RM based policy, respectively. This suggest that if implemented, Reserve policy would open up for criticism as a 'selfish policy' that fulfills $98.24 \%$ for the regional hospital patients, but only manages to address $77.73 \%$ of the networked hospitals' requests. As a public organization with the motto to 'serve society', it is highly unlikely that the health administrator of the regional hospital would consider such a policy. In contrast, the Proportionate policy catered more to external requests and out-performed the three other policies at a $93.35 \%$ fulfillment rate. Naturally, this comes at an expense of the internal emergency and non-emergency requests. When compared with RM policy, a $4.38 \%$ reduction in emergency demand fulfillment was observed, while proportionate policy improve negligible $.65 \%$ external demand request fulfillment.

Finally, we analyzed the proportion of days the regional hospital failed to satisfy all requests for each demand segment. While overall consistent with the two previous metrics this sheds further light on the trade-off between the four policies. Most dramatically it illustrates that the RM and Proportionate policy are more equitable in the sense that they served each demand segment at more balanced rates. In contrast, for Reserve and Sequential we see that the demand segments they favor (emergency and nonemergency, respectively) had a drastically better performance than the other demand streams; specifically, Sequential only failed to fulfill all non-emergency requests .2\% of the time, and Reserve failed to fulfill all emergency requests $1.8 \%$ of the time. In isolation such performance would of course be considered stellar, however, they come at a significant poor performance of fulfilling daily requests for the other demand segments. Specifically, we see that the Reserve and Sequential policies almost half the time were not able to fulfill all external and emergency requests, respectively.

Another interesting and counter-intuitive observation is with regard to the RM policy and the proportion of days it fulfilled all external requests. We see that compared to the Sequential policy the rate decreased by $.88 \%$-points to $75.22 \%$ (days of fulfill all request) for external requests, while the demand fulfillment increased to $92.7 \%$. The reason for this apparent contradiction can be explained by that although the RM policy reserved platelets for the external patients (thus increasing demand fulfillment), it also reserves platelets for the emergency patients. Consequently, there are days where on average, more platelet units available to external demand streams but fewer days external demands will be fully satisfied because a fraction of platelets are reserved for the emergency patients. These results suggest that the RM policy is better suited for handling both emergency cases and for the centralized hospital network, whereas Reserve and Proportionate policies only achieves one.

To summarize, the simulation results substantiated that the RM policy can maximize the benefits for prioritized medically urgent patients, foster an equitable, centralized infrastructure of platelet allocation, while still not significantly increasing platelet wastage due to expiry. Overall, given the supply-constrained context, the RM policy appears to perform better than the other three policies considered. A question though is how robust the results are if the relative availability (supply) of platelets were to increase? Does the RM based policy still perform as well or would the percentage of waste drastically increase (compared to the sequential policy)? To address this 
question, in Sect. 5.4, we extend the simulation analysis by considering different levels of supply. First, to give some insight into why the expiry-rates are so low and how the distribution of waste can be estimated, we provide a numerical illustration of when waste occurs.

\subsection{Likelihood of platelet expiry}

One of the main metrics of interest is the likelihood platelets are wasted due to expiry. In Table 2 we observed that across the four allocation policies the overall percentage of wasted platelet were, despite being small, non-trivial with rates of about .15-.43\%. This motivates to further understand what drives the differences and to derive expressions for each policy's likelihood of platelet expiry. Unfortunately, due to the convoluted stochastic nature of both demand and supply, and the intrinsic inventory consequences of each policy, closed form solutions are mathematically intractable. On the other hand, an ancillary benefit of the simulation analysis is that it enables to numerically estimate any distribution.

As only three day old platelets are going to expire unless used, one would be interested in calculating the probability that the three-day old inventory stock $S_{3, t}$ will not be used. For the hospital's sequential FCFS policy this is relatively easy since it is equivalent to comparing the total aggregated demand distribution with the distribution of $S_{3, t}$. In other words, days when $S_{3, t}>D_{y}+D_{x}+D_{w}$, there is wastage. However, for the RM, Reserve or Proportionate policies, even if the aggregate demand is more than $S_{3, t}$, since platelets are reserved for the later arriving demand streams, there could be wastage. To illustrate, suppose $S_{3, t}=10, D_{w}=8, D_{x}=1, D_{y}=1$, and consider the RM policy with $z_{2}=5$, and $z_{1}=3$. In this case, while total demand equals $S_{3, t}$, only five of the non-emergency request will be fulfilled, and the remaining five platelets are reserved for emergency and external requests, from which only two will be used. Hence, three will expire. Therefore, to approximate the probability of expiry, we instead numerically estimate the distribution of daily fulfilled requests.

The result from one simulation instance is shown in Fig. 6. Each panel, corresponding to each of the four allocation policies, displays the numerical daily distribution of total fulfilled demand (beige) and three day old platelet inventory (turquoise). It is interesting to note that the distribution of the two random variables-total daily fulfilled requests, and daily three day old inventory-exhibit drastic differences in their form depending on the allocation policy. In the figure, we observe that the probability fulfilled demand is less than the three day old inventory is, for all four policies, small. In other words, the region of overlap is small and for the most part the distribution of fulfilled demand is far to the right of the distribution of three day old inventory. This is intuitive since demand is in abundance, while supply is highly constrained.

Based on the numerical distributions, we can approximate the probability when demand will be more than the three day old inventory. The specific values were $99.81 \%$ for the RM policy, and $99.85 \%$ for the sequential FCFS policy. Consequently, the probability of wastage is . $19 \%$ and $.15 \%$ for RM and sequential FCFS, respectively-as observed above. For the Reserve and Proportionate policies the resulting probabilities, regarding when fulfilled demand exceeds three day old inventory, were $99.57 \%$ and 


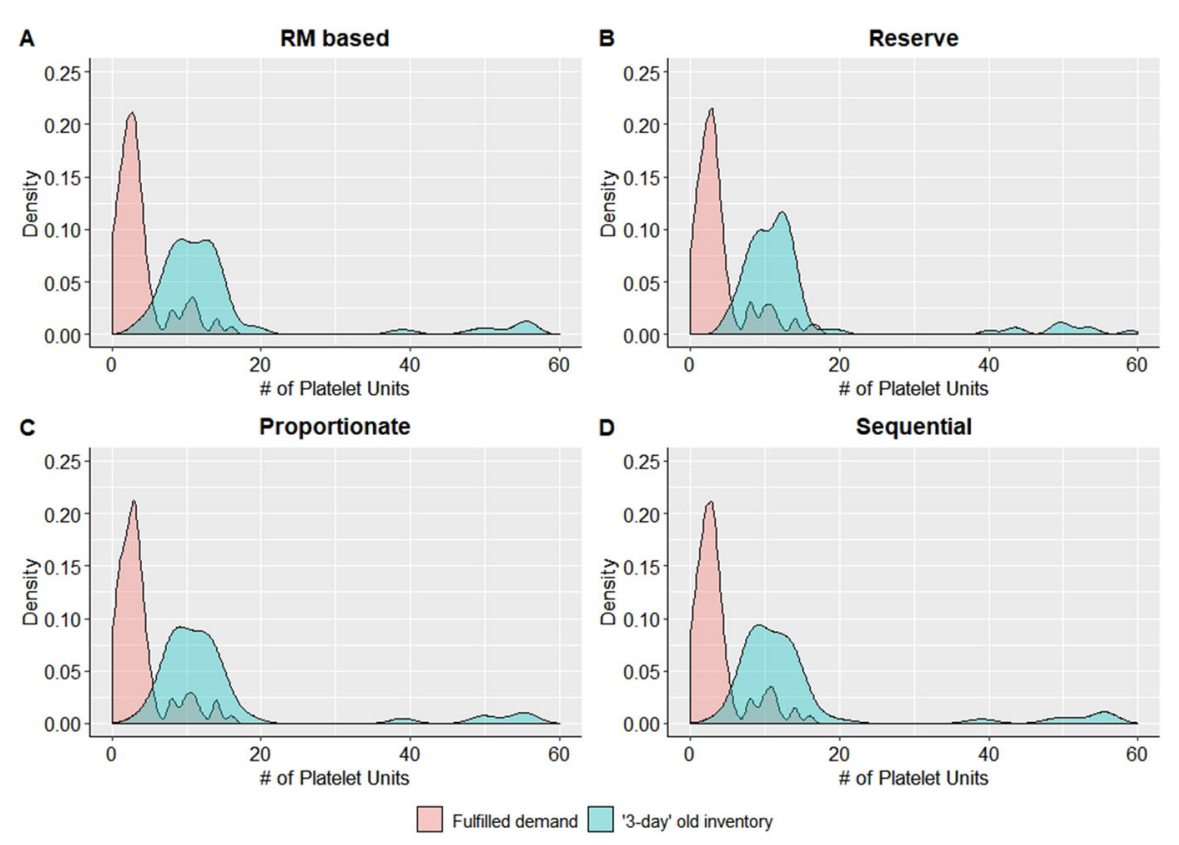

Fig. 6 Plot of fulfilled demand and platelet inventory about to expire ('3-day' old) for a RM based policy, b Reserve policy, c Proportionate policy, d sequential policy

$99.71 \%$. This resulted in a statistically significant increase in platelet expiry when evaluated with sequential policy.

\subsection{Effect of supply on platelet expiry}

Next we investigate if the RM policy would result in a significant worse performance regarding platelet expiry, compared to the sequential policy, if supply is augmented. The motivation of this analysis derives from discussions with the health administrators at the regional hospital, who suggested that the supply levels are (hopefully) expected to improve over the next decade.

To investigate the effect of supply we repeated the above simulation for nine scenarios of the aggregated rate of daily supply under normal day condition. Specifically, we considered a daily rate of $\lambda_{s}=5,6, \ldots, 13$; with the same return process of $\lambda_{r 2}=1$, $\lambda_{r 3}=2$. In other words, the average aggregate supply was varied from 8 to 16 . As point of reference, earlier the mean aggregated daily supply was 10 . For all scenarios, all other parameters were as above, i.e. the mean aggregated daily platelet demand was 12 units, with the segment specific demand rates of $\lambda_{y}=3, \lambda_{x}=7$, and $\lambda_{w}=2$. The main metric of interest is the percentage of waste. The results are presented in Fig. 7.

Figure 7 is a scatter plot of '\% Expiry of platelets' for RM policy ( $x$-axis) versus sequential FCFS policy ( $y$-axis). The solid black line indicates the 45 degree line and represents identical performance between the two policies. There are two interesting 


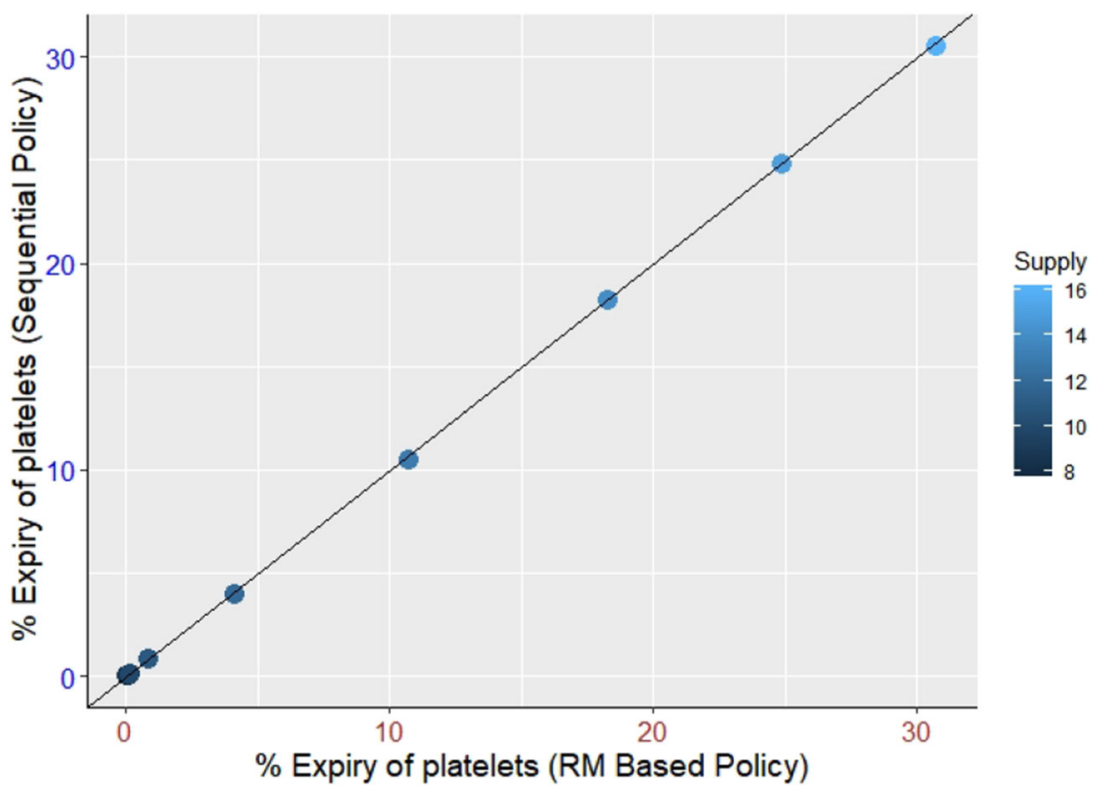

Fig. 7 Policy performance in supply augmented scenario

observations. First, as all observations are virtually on the 45 degree line, the performances of the two policies are essentially identical. That is, even as supply increases the RM policy does not drastically deviate from the sequential policy with respect to wastage. Although not visible in the graph, for all scenarios the RM policy was slightly worse; but never statistically significantly worse. This suggests, that despite a reserve made for emergency and external patients, that won't translate into incremental wastage. This provides solace to the health administrators in emerging economies who might wish to provide more platelet units to the more medically urgent patients but apprehensive about the social responsibility of waste.

Second, when the average total supply is equal or greater than the average total demand, wastage becomes significant, ranging from about $.05 \%$ to $30 \%$. This gives valuable managerial insight into the consequences of balancing supply and demand of platelets, and explains why some western countries experience such a high wastage percentage. In other words, while, on average, having more than sufficient supply of blood platelet will enable a high demand fulfillment, it comes at a high social cost of high proportion of wasted platelets.

\section{Concluding remarks}

Providing safe and sustainable health care is a persistent problem in many developing nations. On the clinical side, developing nations are often plagued by very serious diseases not present in developed nations; prime examples include dengue, malaria and other neglected tropical diseases. Furthermore, on the managerial side, developing 
nations have to make do with less resources-both in absolute and relative terms. As such, health administrators in emerging economies cannot rely on many of the luxuries developed nations have with respect to health care management. A motivating example is access to blood platelets, which in developed nations, as reflected by the extant operations management literature, is in sufficient supply. In sharp and dire contrast, for many emerging economies, not only is demand higher but there is also a continuous shortage and highly volatile supply of blood platelets.

On a positive note, due to concerted efforts of the global community, developing nations have experienced great improvements in healthy living and well-being, social equity and justice, and infrastructure over the past years and decades. A celebrated example includes the UN 2030 Agenda for Sustainable Development, which over the past years has, for instance, assisted with reducing in the incidence of HIV, tuberculosis, hepatitis B, and neglected tropical diseases. ${ }^{7}$ However, despite the positive trends much improvement still remains to be done.

Motivated by the experience at a large regional hospital in north-eastern India in day-today and outbreak situations this paper has analyzed blood platelet allocation considering: (i) stochastic demand from a set of priority differentiated segments, (ii) a highly scarce and stochastic supply, and (iii) the highly perishable nature of blood platelets. The objective has been to derive a simple yet resource efficient allocation policy that balances the aforementioned considerations. Drawing on insight from revenue management, specifically the expected marginal seat revenue heuristic, we developed a near-optimal platelet policy that provides nested allocation quantities in a two-stage decision process. Based on a comprehensive simulation case study, we compared the RM-based policy with the sequential first come, first serve policy, which currently is the practice at the regional hospital, as well as two fixed proportion allocations policies. Overall the RM policy drastically improved the demand fulfillment for the medically more urgent cases while simultaneously not compromising the aggregate demand fulfillment or wastage due to platelet expiry. While trade-offs between different policies is inevitable, the RM policy is furthermore consistent in performance even in dengue outbreak situations. Specifically the emphasis on effective planning of precious resources (i.e. platelets) for improving service capacity and access for medically urgent cases in developing nation is an utmost humanitarian priority (United Nations Statistical Commission 2019). In particular, considering that platelet transfusion is often life-saving replenishment for patients suffering from dengue fever, an infectious neglected tropical disease.

A key parameter to the RM policy is to specify relative 'preference parameters' or 'benefits' from fulfilling the request from the priority differentiated demand segments. On the surface this may appear as a perverse and unethical proposition. After all should not all humans equally valued? Yet in a resource constrained environment, allocation decisions across different patient groups must be made. The social and ethical benefit of the proposed RM policy is that it enables a social planner to set targeted goals with respect to the demand fulfillment of each group, and based on these derive the required parameter values. This is consistent to how public health authorities have established

\footnotetext{
7 Reported in 'Progress of Goal 3 in 2019'; https://sustainabledevelopment.un.org/sdg3, Accessed 20-0319.
} 
targets for various medical processes and treatments. Recently, we have observed priority based ventilator allocation for the COVID-19 patients when the resource (i.e. ventilators) is scarce and demand is far too high. This priority has been determined by health professionals based upon patient health condition and urgency (Baker and Fink 2020). To give another example, in Ontario, Canada, $90 \%$ of patients with complex conditions should in total spend less than eight hours in an emergency room. ${ }^{8}$ In a similar manner the hospital health administrator can in consultation with relevant stakeholders establish benchmark fulfillment rates for each segment that appropriately balances the various medical priorities. To illustrate, suppose a health authority establishes that 90, 80, and 70 percent of all internal emergency, external, and internal non-emergency requests, respectively, should be fulfilled. Based on the specified output results, the input parameters to the RM policy can be calibrated in order to achieve the socially best and most efficient allocation. Furthermore, the relative preference parameter has an intuitive interpretation as it directly corresponds to the critical fractile, i.e. the proportion of demand that should be planned for. Unlike the other fixed proportion allocation policies, the RM policy directly takes into consideration the stochastic nature of future demand. In other words, rather than simply relying on the average future demand, it considers in a near-optimal manner the distribution of future demand. An ancillary benefit is that it provides a mechanism for evaluating if a desired set of output levels is even feasible. For instance, at the case study hospital, while an aggregate demand fulfillment rate of $\mathbf{8 5 - 9 0 \%}$ is possible, it is not possible to achieve such performance for each demand segment.

While the literature on revenue management is vast and with lots of novel applications, and the health care operations management literature is equally large, there has surprisingly been rather modest developments of revenue management applications to health care. As such we hope this study motivates further studies. In particular with respect to scarce health care resource allocation in difficult situations such as sudden demand surge as experienced by different health systems during endemic dengue fever or pandemic COVID-19 outbreaks.

\section{References}

Abbasi, B., Hosseinifard, S.Z.: On the issuing policies for perishable items such as red blood cells and platelets in blood service. Decision Sci. 45(5), 995-1020 (2014)

Abouee-Mehrizi, H., Baron, O., Berman, O., Chen, D.: Managing perishable inventory systems with multiple priority classes. Prod. Oper. Manage. 28(9), 2305-2322 (2019). https://doi.org/10.1111/poms.13058

American Society of Anesthesiologists. Update on Platelets. Technical report (2021). https://www.asahq. org/-/media/sites/asahq/files/public/resources/asa-committees/plate-cobm-asa-final.pdf

Anand, A.: Blood for sale: India's illegal 'red market' (2015). https://www.bbc.com/news/business30273994

Arslan, H., Graves, S.C., Roemer, T.: A single-product inventory model for multiple demand classes. Manage. Sci. 53(9), 1486-1499 (2007)

Ayvaz, N., Huh, W.T.: Allocation of hospital capacity to multiple types of patients. J. Reven. Pricing Manage. 9(5), 386-398 (2010). https://doi.org/10.1057/rpm.2010.30. ISSN 14766930

\footnotetext{
8 For patients with minor conditions the total time should be less than four hours; http://www.health.gov. on.ca/en/pro/programs/waittimes/edrs/targets.aspx, Accessed 20-03-19.
} 
Baker, M., Fink, S.: At the Top of the Covid-19 Curve, How do Hopitals Decide Who Gets Treatment? (2020). https://www.nytimes.com/2020/03/31/us/coronavirus-covid-triage-rationing-ventilators.html

Belobaba, P.P.: Air Travel Demand and Airline Seat Inventory Management. PhD thesis, Massachusetts Institute of Technology (1987)

Belobaba, P.P.: Application of a probabilistic decision model to airline seat inventory control. Oper. Res. 37(2), 183-197 (1989). https://doi.org/10.1287/opre.37.2.183. ISSN 0030364X

Belobaba, P.P.: Optimal vs. heuristic methods for nested seat allocation. In: Presentation at ORSA/TIMS Joint National Meeting (1992)

Bertazzi, L., Paletta, G., Speranza, M.G.: Minimizing the total cost in an integrated vendor-managed inventory system. J. Heuris. 11(5-6 SPEC. ISS.): 393-419 (2005). ISSN 13811231. https://doi.org/10.1007/ s10732-005-0616-6

Blake, J.T., Thompson, S., Smith, S., Anderson, D., Arellano, R., Bernard, D.: Modelling efficiency \& quality in health care. In: Proceedings of 29th Meeting of the EURO Working Group on Operational Research Applied to Health Services, pp. 47-65 (2004)

Blake, J.T., Heddle, N., Barty, R.: Simplified platelet ordering using shortage and outdate targets. Int. J. Health Plann. Manage. 1, 144-156 (2010)

Burgstaler, E.A.: Blood component collection by apheresis. J. Clin. Apher. 21, 142-151 (2006). https://doi. org/10.1002/jca.20043

Chaterjee, P., Tiwari, S.: Patients spend large sums on platelets from private blood banks (2015). https://indianexpress.com/article/cities/delhi/patients-spend-large-sums-on-platelets-fromprivate-blood-banks/

Chen, S., Li, Y., Zhou, W.: Joint decisions for blood collection and platelet inventory control. Prod. Oper. Manage. 28(7), 1674-1691 (2019). https://doi.org/10.1111/poms.13009

Choudhury, N.: Prices of blood units in South East Asia. Asian J. Transf. Sci. 2(2), 45-46 (2008). https:// doi.org/10.4103/0973-6247.42690

Choudhury, N.: Management in Indian blood banking system : true reality. Asian J. Transf. Sci. 3(2), 57-59 (2009). https://doi.org/10.4103/0973-6247.53871

Civelek, I., Karaesmen, I., Scheller-wolf, A.: Blood platelet inventory management with protection levels. Eur. J. Oper. Res. 243(3), 826-838 (2015). https://doi.org/10.1016/j.ejor.2015.01.023. ISSN 03772217

Cushing, M.M.: Platelet Products. In: Shaz, B.H., Hillyer, C.D. (eds.) Transfusion Medicine and Hemostasis, chap 33, 2nd edn, pp 219-226. Newnes (2013). https://doi.org/10.1016/B978-0-12-397164-7.000331

Dawn Karachi: Fixed rates of platelets bags urged (2007). https://www.dawn.com/news/274511

Debroy, S.: No coordination between blood banks and hospitals , 6 lakh litres of blood wasted in five years (2017). https://timesofindia.indiatimes.com/india/no-coordination-between-blood-banks-andhospitals-6-lakh-litres-of-blood-wasted-in-five-years/articleshow/58333338.cms

Ding, Q., Kouvelis, P., Milner, J.: Inventory rationing for multiple class demand under continuous review. Prod. Oper. Manage. 25(8), 1344-1362 (2016). https://doi.org/10.1111/poms.12544

Duan, Q., Liao, T.W.: A new age-based replenishment policy for supply chain inventory optimization of highly perishable products. Int. J. Prod. Econ. 145(2), 658-671 (2013). https://doi.org/10.1016/j.ijpe. 2013.05.020. ISSN 0925-5273

Far, R.M., Rad, F.S., Abdolazimi, Z., Kohan, M.M.D.: Determination of rate and causes of wastage of blood and blood products in Iranian hospitals. Turkish J. Hematol. 31(2), 161-167 (2014). https://doi.org/ 10.4274/tjh.2012.0105

Fletcher, A., Worthington, D.: What is a 'generic' hospital model? A comparison of 'generic' and 'specific' hospital models of emergency patient flows. Health Care Manage. Sci. 12(4), 374-391 (2009). https:// doi.org/10.1007/s10729-009-9108-9. ISSN 13869620

Fontaine, M.J., Chung, Y.T., Rogers, W.M., Sussmann, H.D., Quach, P., Galel, S.A., Goodnough, L.T., Erhun, F.: Improving platelet supply chains through collaborations between blood centers and transfusion services. Transfusion 49(10), 2040-2047 (2009). https://doi.org/10.1111/j.1537-2995.2009. 02236.x

Frank, K.C., Zhang, R.Q., Duenyas, I.: Optimal policies for inventory systems with priority demand classes. Oper. Res. 51(6), 993-1003 (2003). https://doi.org/10.1287/opre.51.6.993.24923. ISSN 0030364X

Haijema, R., van der Wal, J., van Dijk, N.M.: Blood platelet production: a multi-type perishable inventory problem. In: Operations Research Proceedings, 2004, pp 84-92. Springer, Berlin, Heidelberg (2005). ISBN 3540242740 
Haijema, R., van der Wal, J., van Dijk, N.M.: Blood platelet production: optimization by dynamic programming and simulation. Comput. Oper. Res. 34(3), 760-779 (2007). https://doi.org/10.1016/j.cor.2005. 03.023

Haijema, R., van Dijk, N., van der Wal, J., Sibinga, C.: Blood platelet production with breaks: optimization by SDP and simulation. In. J. Prod. Econ. 121(2), 464-473 (2009). https://doi.org/10.1016/j.ijpe.2006. 11.026

Horstick, O., Tozan, Y., Wilder-smith, A.: Reviewing dengue: still a neglected tropical disease? PLoS Neglect. Tropi. Dis. 9(4) (2015). https://doi.org/10.1371/journal.pntd.0003632

Jain, P.: Platelet cost shoots up as dengue cases rise in Bhopal (2016). https://www. hindustantimes.com/bhopal/platelet-cost-shoots-up-as-dengue-cases-rise-in-bhopal/storybKJghyN4wVVtODNA0iUI6H.html

Kamath, V.: The Burden of Recovery: Dengue Can Cost You Big (2016). https://bangaloremirror. indiatimes.com/bangalore/others/the-burden-of-recovery-dengue-can-cost-you-big/articleshow/ $52770355 . \mathrm{cms}$

Law, A.M., Kelton, W.D.: Simulation Modeling and Analysis. McGraw-Hill Higher Education, New York, NY (2000), https://doi.org/10.1145/1667072.1667074

Littlewood, K.: Forecasting and control of passenger bookings. In: Airline Group International Federation of Operational Research Societies Proceedings, pp. 95-117 (1972)

Mishra, L.: Five-fold increase in cost of blood platelets with spike in dengue cases (2014). https:// mumbaimirror.indiatimes.com/mumbai/civic/five-fold-increase-in-cost-of-blood-platelets-withspike-in-dengue-cases/articleshow/45040317.cms

National Blood Authority Australia. National Blood and Blood Product Wastage Reduction Strategy 2013-2017. Technical report (2013). https://www.blood.gov.au/system/files/documents/nbawastage-strategy.pdf

Paul, A., Rajapakshe, T., Mallik, S.: Socially optimal contracting between a regional blood bank and hospitals. Prod. Oper. Manage. 28(4), 908-932 (2019). https://doi.org/10.1111/poms.12960

Petraszko, T., Zeller, M.: Chapter 18: Platelet transfusion, alloimmunization and management of platelet refractoriness. In: Clinical Guide to Transfusion, pp. 1-18 (2018)

Prastacos, G.P.: Blood inventory management: an overview of theory and practice. Manage. Sci. 30(7), 777-800 (1984)

Raman, S.: Illicit India 'blood Farm’ Raided (2008). http://news.bbc.co.uk/2/hi/south_asia/7302649.stm

Ravishanker, R.: Platelet Transfusion Remains a Costly Affair (2017). http://www.newindianexpress.com/ cities/bengaluru/2017/aug/08/platelet-transfusion-remains-a-costly-affair-1640048.html

Shetty, D.: Needed! Blood Platelets Across City Blood Banks (2016). https://www.dnaindia.com/mumbai/ report-needed-blood-platelets-across-city-blood-banks-2262244

Singh, J.: Arrests in India 'Blood Scandal' (2009). http://news.bbc.co.uk/2/hi/south_asia/8103124.stm

United Nations Statistical Commission. Global Indicator Framework for the Sustainable Development Goals and Targets of the 2030 Agenda for Sustainable Development. Technical report (2019). https://unstats. un.org/sdgs/indicators/indicators-list

Wang, H., Liang, X., Sethi, S., Yan, H.: Inventory commitment and prioritized backlogging clearance with alternative delivery lead times. Prod. Oper. Manage. 23(7), 1227-1242 (2014). https://doi.org/10. 1111/poms.12162. ISSN 19375956

Whitaker, B.I., Sullivan, M.: The 2005 Nationwide Blood Collection and Utilization Survey Report. Technical report, US Department of Health and Human Services, Washington, DC (2005)

WHO. Manual on the Management, Maintenance and Use of Blood Cold Chain Equipment. Technical report (2005)

Zhang, R.Q., Hopp, W.J., Supatgiat, C.: Spreadsheet implementable inventory control for a distribution center. J. Heurist. 7(2), 185-203 (2001). https://doi.org/10.1023/A:1009613921001. ISSN 13811231

Zhou, D., Leung, L.C., Pierskalla, W.P.: Inventory management of platelets in hospitals: optimal inventory policy for perishable products with regular and optional expedited replenishments. Manufact. Serv. Oper. Manage. 13(4), 420-438 (2011)

Publisher's Note Springer Nature remains neutral with regard to jurisdictional claims in published maps and institutional affiliations. 